PROCEEDINGS OF THE

AMERICAN MATHEMATICAL SOCIETY

Volume 127, Number 6, Pages 1583-1588

S 0002-9939(99)04732-2

Article electronically published on February 17, 1999

\title{
BINOMIAL FREE RESOLUTIONS FOR NORMAL TORIC SURFACES
}

\author{
VESSELIN GASHAROV AND IRENA PEEVA
}

(Communicated by Wolmer V. Vasconcelos)

\begin{abstract}
We construct the minimal free resolution of the residue field over a normal toric surface.
\end{abstract}

\section{INTRODUCTION}

Any normal toric surface is given by a normal 2-dimensional submonoid $\Lambda$ of $\mathbb{N}^{2}$ (cf. $[\mathrm{Fu}]$ ). Denote by $\alpha_{1}, \ldots, \alpha_{n}$ the minimal generators of $\Lambda$. Then

$$
k[\Lambda] \cong k\left[x_{1}, \ldots, x_{n}\right] / I_{\Lambda},
$$

where $I_{\Lambda}$ is the toric ideal equal to the kernel of the map $k\left[x_{1}, \ldots, x_{n}\right] \rightarrow k\left[z_{1}, z_{2}\right]$ sending $x_{i}$ to $\mathbf{z}^{\alpha_{i}}=z_{1}^{\alpha_{i 1}} z_{2}^{\alpha_{i 2}}$. The ideal $I_{\Lambda}$ might not be homogeneous with respect to the usual grading, but it is always $\mathbb{N}^{2}$-graded.

We are interested in resolving $k$ as a $k[\Lambda]$-module. The Betti numbers were first studied in [LS], and then it was proved in [PRS] (also see [HRW] ) that

$$
\operatorname{dim}_{k} \operatorname{Tor}_{i}^{k[\Lambda]}(k, k)=(n-2)^{i-2}(n-1)^{2} \quad \text { for } i \geq 2 .
$$

In this paper we construct a minimal free resolution $\mathbb{F}$ of the residue field $k$ over $k[\Lambda]$. The resolution has the following properties:

- the basis elements of $\mathbb{F}$ as a $k[\Lambda]$-module and the differential maps are given explicitly by simple formulas;

- the resolution $\mathbb{F}$ is binomial: for $i \geq 2$ denote by $D_{i}$ the matrix of the differential $d_{i}$ in $\mathbb{F}$, then any column in $D_{i}$ has two non-zero entries and they are monomials;

- $\mathbb{F}$ lifts the minimal free resolution in [Fr, Theorem in $\S 3]$ of $k$ over $k\left[x_{1}, \ldots, x_{n}\right] /$ $i n_{\prec}\left(I_{\Lambda}\right)$, where $i n_{\prec}\left(I_{\Lambda}\right)$ is a quadratic initial ideal.

The construction of $\mathbb{F}$ is presented in Section 2 and is proved in Section 3. We demonstrate the construction in Example 2.5 for the twisted cubic curve; in this very special example minimal free resolutions of $k$ are given by Golod's construction, Priddy's construction, and Anick's construction, however none of them is as simple as $\mathbb{F}$. The motivation for this paper was to obtain explicit nice minimal free resolutions for all rational normal curves.

Received by the editors September 4, 1997.

1991 Mathematics Subject Classification. Primary 13D02.

(C)1999 American Mathematical Society 


\section{Minimal fReE RESOLUtion}

In this section we construct the minimal free resolution of $k$ over a normal toric surface and demonstrate the construction in Example 2.5.

Order the unique set of minimal generators $\alpha_{1}, \ldots, \alpha_{n}$ of $\Lambda$ so that $\operatorname{det}\left(\alpha_{i}, \alpha_{i+1}\right) \leq$ 0 for $i=1,2, \ldots, n-1$. Let $\prec$ be the purely lexicographic term order on $k\left[x_{1}, \ldots, x_{n}\right]$. The toric ideal $I_{\Lambda}$ has a minimal Gröbner basis consisting of the $\left(\begin{array}{c}n-1 \\ 2\end{array}\right)$ binomials:

$$
\underline{x_{i} x_{i+j}}-x_{i+1} x_{i+j-1} \prod_{p=i+1}^{i+j-1} x_{p}^{b_{p}}, \quad 1 \leq i \leq n-2,2 \leq j \leq n-i .
$$

(For definition and properties of Gröbner basis see [Ei].) The underlined monomials generate the initial ideal $i n_{\prec}\left(I_{\Lambda}\right)=\left(x_{i} x_{j}: 1 \leq i<j-1 \leq n-1\right)$, which is the Stanley-Reisner ideal for the "zig-zag poset" poset $P$ on $\left\{x_{1}, x_{2}, \ldots, x_{n}\right\}$ (namely, $P$ has covering relations $x_{i}<_{P} x_{i+1}$ if $i$ is odd and $x_{i}>_{P} x_{i+1}$ if $i$ is even).

A minimal free resolution of $k$ over $k\left[x_{1}, \ldots, x_{n}\right] / i n_{\prec}\left(I_{\Lambda}\right)$ is given in [Fr, Theorem in §3]. Our construction lifts this resolution to $k[\Lambda]$ using the specific relations (2.1).

We now construct a minimal free resolution $\mathbb{F}$ of $k$ over $k[\Lambda]$. We define $\mathbb{F}$ to be the free $k[\Lambda]$-module $k[\Lambda] \otimes_{k} Q$, where $Q$ is the non-commutative $k$-algebra

$$
Q=k\left\langle y_{1}, \ldots, y_{n}\right\rangle /\left(\left\{y_{i} y_{i+1}+y_{i+1} y_{i}\right\}_{1 \leq i \leq n-1},\left\{y_{j}^{2}\right\}_{1 \leq j \leq n}\right) .
$$

As we mentioned in the introduction, by [PRS] the Betti numbers of $k$ are

$$
\operatorname{dim}_{k} \operatorname{Tor}_{i}^{k[\Lambda]}(k, k)=(n-2)^{i-2}(n-1)^{2} \quad \text { for } i \geq 2 .
$$

So the Poincaré series of $k$ is

$$
\frac{(1+t)^{2}}{1-(n-2) t}
$$

and it can be seen by $[\mathrm{Fr}]$ that the Hilbert series of $Q$ is the same. We say that a $y$-monomial $y_{i_{1}} y_{i_{2}} \ldots y_{i_{p}} \in Q$ is standard if $y_{i_{1}}$ is the smallest variable that can be factored to the left (modulo the relations defining $Q$ ) and $y_{i_{2}} \ldots y_{i_{p}}$ is standard. The standard $y$-monomials of degree $i$ form a basis for the free $k[\Lambda]$-module $\mathbb{F}_{i}$. In what follows the letter $m$ denotes a standard $y$-monomial.

Next we define a differential $d$ on $\mathbb{F}$ which is homogeneous with respect to the $\mathbb{N}^{2}$-grading. For $y$-monomials of degree $\leq 2$ we set

$$
\begin{aligned}
d\left(y_{s}\right) & =x_{s}, \\
d\left(y_{i} y_{i+1}\right) & =-d\left(y_{i+1} y_{i}\right)=x_{i} y_{i+1}-x_{i+1} y_{i}, \\
d\left(y_{i} y_{i+j}\right) & =x_{i} y_{i+j}-c_{i j} y_{i+j-1}, \\
d\left(y_{i+j} y_{i}\right) & =x_{i+j} y_{i}-c_{i j}^{\prime} y_{i+1},
\end{aligned}
$$

where $1 \leq s \leq n, 1 \leq i \leq n-1$, and $2 \leq j \leq n-i$. The coefficients $c_{i j}=$ $x_{i+1} \prod_{p=i+1}^{i+j-1} x_{p}^{\overline{b_{p}}}$ and $c_{i j}^{\prime}=x_{i+j-1} \prod_{p=i+1}^{i+j-1} x_{p}^{b_{p}}$ are uniquely determined by $(2.1)$; this choice of the coefficients ensures that the differential is homogeneous with respect to the $\mathbb{N}^{2}$-grading.

Let $y_{i} y_{j} y_{l} m$ be an arbitrary standard $y$-monomial of degree $\geq 3$. We define

$$
d\left(y_{i} y_{j} y_{l} m\right)= \begin{cases}d\left(y_{i} y_{l}\right) y_{j} m & \text { if } i>l=j+1 \\ d\left(y_{i} y_{j}\right) y_{l} m & \text { otherwise. }\end{cases}
$$


Finally we extend the action of the differential $d$ to all of $\mathbb{F}$ by $k[\Lambda]$-linearity. The following theorem is our main result; it is proved in the next section.

Theorem 1. $(\mathbb{F}, d)$ is a minimal free resolution of $k$ over $k[\Lambda]$.

Example 2.5. The twisted cubic curve. We consider the monoid $\Lambda$ generated by $\alpha_{1}=(0,3), \alpha_{2}=(1,2), \alpha_{3}=(2,1), \alpha_{4}=(3,0) \in \mathbb{N}^{2}$. The monoid algebra

$$
k[\Lambda] \cong k\left[x_{1}, \ldots, x_{4}\right] /\left(x_{1} x_{3}-x_{2}^{2}, x_{2} x_{4}-x_{3}^{2}, x_{1} x_{4}-x_{2} x_{3}\right)
$$

is the toric ring of the twisted cubic curve. In this case the equations in (2.1) are the defining equations. The minimal free resolution of $k$ over $k[\Lambda]$ is

$$
\mathbb{F}=k[\Lambda] \otimes k\left\langle y_{1}, \ldots, y_{4}\right\rangle /\left(\left\{y_{i} y_{i+1}+y_{i+1} y_{i}\right\}_{1 \leq i \leq 3},\left\{y_{i}^{2}\right\}_{1 \leq i \leq 4}\right) .
$$

The differential acts on the standard monomials in the following way (the notation is as in (2.2) and (2.3)):

$$
\begin{gathered}
d\left(y_{i}\right)=x_{i}, \\
d\left(y_{i} y_{i+1}\right)=x_{i} y_{i+1}-x_{i+1} y_{i}, \\
d\left(y_{1} y_{3}\right)=x_{1} y_{3}-x_{2} y_{2}, \quad d\left(y_{2} y_{4}\right)=x_{2} y_{4}-x_{3} y_{3}, \quad d\left(y_{1} y_{4}\right)=x_{1} y_{4}-x_{2} y_{3}, \\
d\left(y_{3} y_{1}\right)=x_{3} y_{1}-x_{2} y_{2}, \quad d\left(y_{4} y_{2}\right)=x_{4} y_{2}-x_{3} y_{3}, \quad d\left(y_{4} y_{1}\right)=x_{4} y_{1}-x_{3} y_{2}, \\
d\left(y_{3} y_{1} y_{2} m\right)=d\left(y_{3} y_{2}\right) y_{1} m, \\
d\left(y_{4} y_{1} y_{2} m\right)=d\left(y_{4} y_{2}\right) y_{1} m, \\
d\left(y_{4} y_{2} y_{3} m\right)=d\left(y_{4} y_{3}\right) y_{2} m, \\
d\left(y_{i} y_{j} y_{l} m\right)=d\left(y_{i} y_{j}\right) y_{l} m \text { otherwise. }
\end{gathered}
$$

\section{ProOF}

In this section we prove Theorem 2.4 in a sequence of three lemmas:

Theorem 2. $(\mathbb{F}, d)$ is a complex.

Proof. In order to simplify the notation we consider $(\overline{\mathbb{F}}, \bar{d})=(\mathbb{F}, d) /\left(\left\{x_{i}-1\right\}_{1 \leq i \leq n}\right)$. Since $(\mathbb{F}, d)$ is multigraded and $k[\Lambda]$ is one-dimensional (over $k$ ) in each multidegree, it suffices to show that $\bar{d}^{2}$ annihilates all standard $y$-monomials. The action of $\bar{d}$ on a standard $y$-monomial $M$ depends only on the arrangement of the three leftmost variables in $M$. Our proof splits into seven possible cases for these arrangements.

Case 1. If $M$ has degree $\leq 2$, then $\bar{d}^{2}(M)=0$ by (2.2) and the relations (2.1).

In the remaining cases $\operatorname{deg}(M) \geq 3$ and $i, j, q, p$ are suitable positive integers.

Case 2. Let $M=y_{i} y_{i+j} y_{i+j+q} m$. Then

$$
\begin{aligned}
\bar{d}^{2}(M) & =\bar{d}\left(y_{i+j} y_{i+j+q} m-y_{i+j-1} y_{i+j+q} m\right) \\
& =y_{i+j+q} m-y_{i+j+q-1} m-y_{i+j+q} m+y_{i+j+q-1} m=0 .
\end{aligned}
$$

Case 3. Let $M=y_{i} y_{i+j} y_{i+j-2} m$. Note that $y_{i+j-1}$ cannot be the first variable of $m$ since it commutes with $y_{i+j} y_{i+j-2}$ and $M$ is standard. Hence

$$
\begin{aligned}
\bar{d}^{2}(M) & =\bar{d}\left(y_{i+j} y_{i+j-2} m-y_{i+j-1} y_{i+j-2} m\right) \\
& =y_{i+j-2} m-y_{i+j-1} m+y_{i+j-1} m-y_{i+j-2} m=0 .
\end{aligned}
$$


Case 4. Let $M=y_{i} y_{i+j} y_{i+j-p} m$, where $p \geq 3$. Then

$$
\bar{d}^{2}(M)=\bar{d}\left(y_{i+j} y_{i+j-p} m-y_{i+j-1} y_{i+j-p} m\right) .
$$

This evaluates to $y_{i+j-p} m-y_{i+j-p+1} m-y_{i+j-p} m+y_{i+j-p+1} m=0$ if $m$ does not start with $y_{i+j-p+1}$. Otherwise we write $m=y_{i+j-p+1} \tilde{m}$ and

$$
\bar{d}^{2}(M)=\bar{d}\left(y_{i+j} y_{i+j-p+1}\right) y_{i+j-p} \tilde{m}-\bar{d}\left(y_{i+j-1} y_{i+j-p+1}\right) y_{i+j-p} \tilde{m}=0 .
$$

Case 5. Let $M=y_{i+j} y_{i} y_{i+q} m$, where $q \geq 2$. Then

$$
\begin{aligned}
\bar{d}^{2}(M) & =\bar{d}\left(y_{i} y_{i+q} m-y_{i+1} y_{i+q} m\right) \\
& =y_{i+q} m-y_{i+q-1} m-y_{i+q} m+y_{i+q-1} m=0 .
\end{aligned}
$$

Case 6. Let $M=y_{i+j} y_{i} y_{i+1} m$, where $j \geq 2$. Here $m$ cannot start with $y_{i+1}$; therefore

$$
\begin{aligned}
\bar{d}^{2}(M) & \left.=\bar{d}\left(\bar{d}\left(y_{i+j} y_{i+1}\right) y_{i} m\right)\right) \\
& =\bar{d}\left(y_{i+1} y_{i} m-y_{i+2} y_{i} m\right) \\
& =y_{i} m-y_{i+1} m-y_{i} m+y_{i+1} m=0 .
\end{aligned}
$$

Case 7. Let $M=y_{i+j} y_{i} y_{i-p} m$, where $p \geq 2$. Note that if $p=2$, then $m$ cannot start with $y_{i-1}$ because $y_{i-1}$ commutes with $y_{i} y_{i-2}$ and $M$ is standard. We have $\bar{d}^{2}(M)=\bar{d}\left(y_{i} y_{i-p} m-y_{i+1} y_{i-p} m\right)$. This evaluates to $y_{i-p} m-y_{i-p+1} m-y_{i-p} m+$ $y_{i-p+1} m=0$ if $m$ does not start with $y_{i-p+1}$. Otherwise write $m=y_{i-p+1} \tilde{m}$ and

$$
\bar{d}^{2}(M)=\bar{d}\left(y_{i} y_{i-p+1}\right) y_{i-p} \tilde{m}-\bar{d}\left(y_{i+1} y_{i-p+1}\right) y_{i-p} \tilde{m}=0 .
$$

The complex $\mathbb{F}$ is spanned as a $k$-vector space by monomials in $x$ and $y$. The commutative $x$-part obeys the relations in $k[\Lambda]$ and the non-commutative $y$-part obeys the relations in $Q$. We call such a monomial spanning if it has the form $x_{i}^{c} x_{i+1}^{e} y_{i+1} y_{j} m$ where either $y_{i+1} y_{j} m$ is standard or $i=j$ and $y_{i} y_{i+1} m$ is standard.

Theorem 3. The set of spanning monomials spans the $k$-vector space $\mathbb{F} / \operatorname{Im}(d)$.

Proof. Every monomial in $\mathbb{F}_{\geq 2}$ can be written in the form $M=x_{i}^{c} x_{i+1}^{e} y_{r} y_{s} m$, where $y_{r} y_{s} m$ is standard. If $c=e=0$, then $M$ is spanning. We therefore assume $c>0$. We set $X(M)=i$ and $Y(M)=r$. Thus $M$ is spanning if and only if $Y(M)-X(M)=1$. We will show that every non-spanning monomial $M$ is congruent modulo $\operatorname{Im}(d)$ to another monomial $M^{\prime}$ which is closer to being spanning. Depending on whether $X(M)-Y(M)$ is positive, zero, or negative, we use one of the following reduction procedures:

Negative reduction: Suppose $M=x_{i}^{w} x_{i+1}^{v} y_{i+j} y_{z} m$ with $j \geq 2$. Set

$$
M^{\prime}=M-d\left(x_{i}^{w-1} x_{i+1}^{v} y_{i} y_{i+j} y_{z} m\right) .
$$

Rewriting $M^{\prime}$ in standard form, we see that $Y(M)>Y\left(M^{\prime}\right)$ and $X(M) \leq X\left(M^{\prime}\right)$. Thus $M^{\prime}$ is congruent to $M$ modulo $\operatorname{Im}(d)$ and $Y(M)-X(M)>Y\left(M^{\prime}\right)-X\left(M^{\prime}\right)$. Positive reduction 1: Suppose $M=x_{i+j}^{w} x_{i+j+1}^{v} y_{i} y_{z} m, z \neq i+1$ and $j>0$. Set $M^{\prime}=M-d\left(x_{i+j}^{w-1} x_{i+j+1}^{v} y_{i+j} y_{i} y_{z} m\right)$. Rewriting $M^{\prime}$ in standard form, we find $Y(M)<Y\left(M^{\prime}\right)$ and $X(M) \geq X\left(M^{\prime}\right)$, hence $X(M)-Y(M)>X\left(M^{\prime}\right)-Y\left(M^{\prime}\right)$. 
Positive reduction 2: Suppose $M=x_{i+j}^{w} x_{i+j+1}^{v} y_{i} y_{i+1} m$ with $j>0$. Set $M^{\prime}=$ $-M-d\left(x_{i+j}^{w-1} x_{i+j+1}^{v} y_{i+j} y_{i+1} y_{i} m\right)$. Then $X(M)-Y(M)>X\left(M^{\prime}\right)-Y\left(M^{\prime}\right)$.

Zero reduction: Suppose $M=x_{i}^{w} x_{i+1}^{v} y_{i} y_{z} m$, where $v>0$ and $z \neq i+1$. Then

$$
M^{\prime}=d\left(x_{i}^{w} x_{i+1}^{v-1} y_{i} y_{i+1} y_{z} m\right)+M=x_{i}^{w+1} x_{i+1}^{v-1} y_{i+1} y_{z} m
$$

is a spanning monomial.

Starting with any monomial $M$ in $\mathbb{F}_{\geq 2}$, we can use a sequence of reductions as above to replace $M$ by a spanning monomial modulo $\operatorname{Im}(d)$.

Theorem 4. The complex $(\mathbb{F}, d)$ is exact.

Proof. Consider any non-zero $\Lambda$-homogeneous $k$-linear combination of spanning monomials,

$$
N=\sum_{s} \beta_{s} x_{q_{s}}^{w_{s}} x_{q_{s}+1}^{v_{s}} y_{q_{s}+1} y_{z_{s}} m_{s},
$$

where $\beta_{s} \in k$. To prove Lemma 3.3, we must show that $d(N) \neq 0$. We shall assume that each monomial in $N$ is written so that the differential acts on the first two $y$-variables (to obtain this we switch the second and third $y$-variables if necessary). We call $z_{s}$ the order of the term $x_{q_{s}}^{w_{s}} x_{q_{s}+1}^{v_{s}} y_{q_{s}+1} y_{z_{s}} m_{s}$. Setting $u=\min \left\{z_{s}\right\}$, we can write

$$
N=\beta x_{q}^{w} x_{q+1}^{v} y_{q+1} y_{u} m+\text { terms of order } \geq u,
$$

where $\beta \in k$. Since $k[\Lambda]$ is one-dimensional in each multidegree, $N$ is multihomogeneous, and each term of $N$ is spanning, we find that there is only one term in $N$ which has order $u$ and ends on $m$.

Suppose that $d(N)=0$. The term $L=c x_{q}^{w} x_{q+1}^{v+1} y_{u} m$ appears in $d(N)$ and must cancel. Since $N$ contains no spanning monomials of order $u-1$, a scalar multiple of $L$ must appear in $d\left(x_{h}^{f} x_{h+1}^{g} y_{h+1} y_{u+1} m\right)$ for some $f, g, h$. This is only possible if $u>q \geq h$, and in view of $y_{q+1} y_{u} \neq 0$, we conclude that $u \geq q+2$. But then the spanning monomial $c x_{q}^{w} x_{q+1}^{v} y_{q+1} y_{u} m$ contributes a term $p y_{u-1} m$ (with $p$ a monomial in the $x$-variables) to the expansion of $d(N)=0$. This term $p y_{u-1} m$ appears in the differential of another spanning monomial from $N$. Such a spanning monomial must have order $u$ and end on $m$. Hence $N$ contains two or more distinct spanning monomials of order $u$ and ending on $m$. This is a contradiction. Thus, $(\mathbb{F}, d)$ is exact.

This completes the proof of Theorem 2.4 since $(\mathbb{F}, d)$ is minimal by construction (the entries in the matrices of the differential maps are in the ideal $\left(x_{1}, \ldots, x_{n}\right)$ ).

\section{REFERENCES}

[Ei] D. Eisenbud, Commutative Algebra With a View Toward Algebraic Geometry, SpringerVerlag, NY, 1995. MR 97a:13001

[Fu] W. Fulton, Introduction to toric varieties, Ann. Math. Studies 131, Princeton University Press, Princeton, 1993. MR 94g:14028

[HRW] J. Herzog, V. Reiner, and V. Welker, The Koszul property in affine semigroup rings, preprint (1997).

[Fr] R. Fröberg, Determination of a class of Poincaré series, Math. Scand. 37 (1975), 29-39. MR 53:8057 
[LS] O. Laudal and A. Sletsjøe, Betti numbers of monoid algebras. Applications to 2dimensional torus embeddings, Math. Scand. 56 (1985), 145-162. MR 87h:13010

[PRS] I. Peeva, V. Reiner, and B. Sturmfels, How to shell a monoid, preprint, Math. Ann. 310 (1998), 379-393. CMP 98:07

Department of Mathematics, University of Michigan, Ann Arbor, Michigan 48109 Current address: Department of Mathematics, Cornell University, Ithaca, New York 14853

Department of Mathematics, Massachusetts Institute of Technology, Cambridge, MASSACHUSETTS 02139

Current address: Department of Mathematics, Cornell University, Ithaca, New York 14853 